\title{
含微量过渡元素的铁基纳米晶 合金性能的研究
}

\author{
刘学东王景唐
}

(中国科学院金属研究所, 沈阳 110015)

\section{关锱调纳米晶合金、显微硬度、Hall-Petch 关系}

近年来, 具有纳米级晶粒尺寸的多晶材料的研究引起了人们极大的兴趣. 很重要的原因 是晶界已成为该材料中一项不可乎略的组元. 这些材料中大量晶界的存在导致许多与微结构 特性有关的性能发生变化,如反常 Hall-Petch 关系的发现 ${ }^{[1,2]}$. Lu 等 ${ }^{[3]}$ 提出了利用非晶晶化 法制备大块纳米晶合金，即通过控制热处理工艺使非晶条带、丝或粉晶化成具有一定晶粒尺寸 的纳米晶材料，为直接生产大块纳米晶合金提供了可能性，本文利用该方法成功地制备了晶 粒尺寸在 15-250nm 范围内的 Fe-TM-Si-B (TM - Cu, Mo) 多晶合金, 并研究了显微硬度

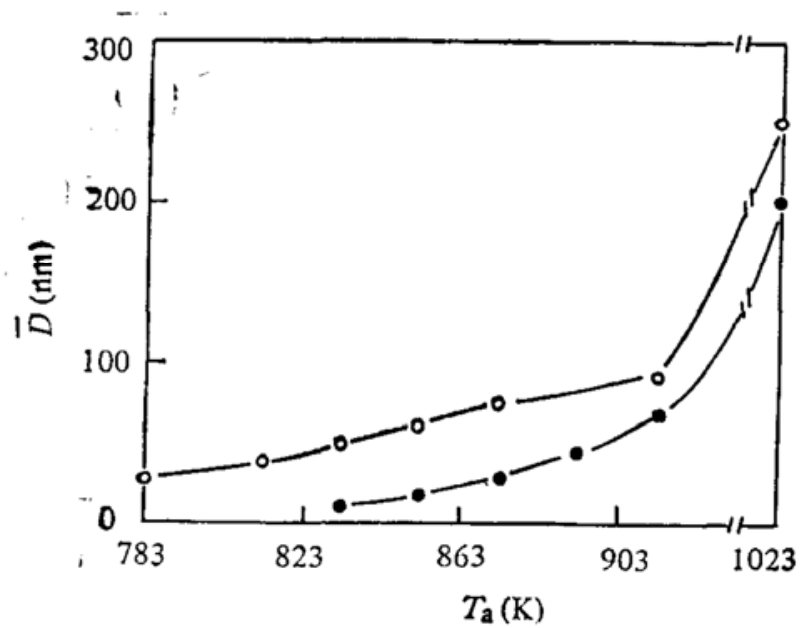

图 $1 \mathrm{Fe}-\mathrm{Cu}-\mathrm{Si}-\mathrm{B}(\mathrm{O})$ 及 $\mathrm{Fe}-\mathrm{Mo}-\mathrm{Si}-\mathrm{B}(\bullet)$ 合金 平均晶粒尺寸 $\bar{D}$ 随退火温度 $T_{\mathrm{a}}$ 的变化

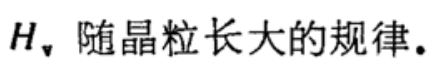

非晶 $\left(\mathrm{Fe}_{0.99} \mathrm{TM}_{0.01}\right)_{78} \mathrm{Si}_{9} \mathrm{~B}_{13}$ (at \% ), $\mathrm{TM}=$ $\mathrm{Cu}, \mathrm{Mo}$ 条带采用单辊急冷法制备. 退火态 样品的晶化相及晶粒度分别在 Rigaku X 射 线衍射仪和 Philips EM 420 分析电镜上确

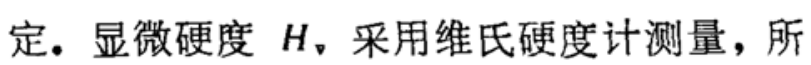
用砝码为 $50 \mathrm{~g}$, 每次测量时间为 $10 \mathrm{~s}$.

经 DSC 扫描知 (升温速度为 $20 \mathrm{~K} / \mathrm{min}$ ), 含 $\mathrm{Cu}$ 非晶合金的晶化温度较含 Mo 非晶合 金的值低, 分别为 $\mathrm{Fe}-\mathrm{Cu}-\mathrm{Si}-\mathrm{B}: T_{\mathrm{pl}}-758.4$ $\mathrm{K}, T_{\mathrm{p} 2}=826.6 \mathrm{~K} ; \mathrm{Fe}-\mathrm{Mo}-\mathrm{Si}-\mathrm{B}: T_{\mathrm{p} 1}=841.0$ $\mathrm{K}, T_{\mathrm{p} 2}=858.0 \mathrm{~K}$; 将两组非晶条带在 783 $1023 \mathrm{~K}$ 温度范围内等温退火 $1 \mathrm{~h}$ 后可得到不 同晶粒尺寸的多晶合金,结果示于图 1. 图 2 分别为两组样品在 $783 \mathrm{~K}$ 和 $833 \mathrm{~K}$ 等温退 火 $1 \mathrm{~h}$ 的 TEM 图象. 显然, 两组样品经退火后均已完全晶化, 晶化组织均匀, 无枝晶生成.

多晶材料的显微硬度 $H_{\mathrm{v}}$. 随晶粒长大的变化示于图 3. 可见, 对于 $\mathrm{Fe}-\mathrm{Cu}-\mathrm{Si}-\mathrm{B}$ 合金而 言, 随着晶粒尺寸的增大, 显微硬度 $H$ 、随之下降. 但上述规律并不适用于 $\mathrm{Fe}-\mathrm{Mo}-\mathrm{Si}-\mathrm{B}$ 合 金. 该合金在平均晶粒尺寸为 $45 \mathrm{~nm}$ 时, $H_{\text {、 }}$ 取得极大值, $\left(H_{\mathrm{v}}\right)_{\max }-10.0 \pm 0.3 \mathrm{GPa}$. 当晶 粒尺寸大于此值时, 晶粒的长大使合金的硬度明显下降. 但当平均晶粒尺寸小于此值时, $H_{\mathrm{v}}$ 却有相反的变化怣律. 因此, Fe-Mo-Si-B 多晶材料的 $H$ 、变化具有临界现象, 临界点为 $45 \mathrm{~nm}$.

1992-09-04 收稿, 1992-11-18 收修改稿. 




图 2 纳米晶合金的 TEM 图象

(a) $\mathrm{Fe}-\mathrm{Mo}-\mathrm{Si}-\mathrm{B}(333 \mathrm{~K} \times 1 \mathrm{~h})$, (b) $\mathrm{Fe}-\mathrm{Cu}-\mathrm{Si}-\mathrm{B}(783 \mathrm{~K} \times 1 \mathrm{~h})$

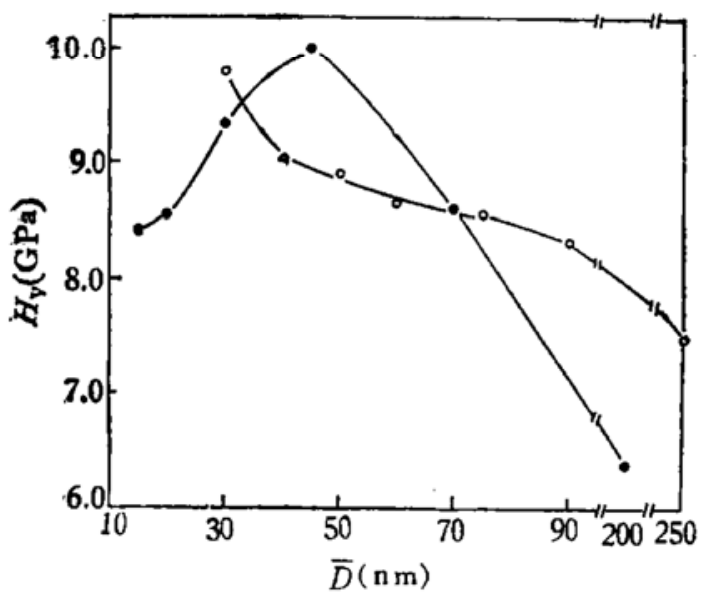

图 $3 \mathrm{Fe}-\mathrm{Cu}-\mathrm{Si}-\mathrm{B}(0)$ 及 $\mathrm{Fe}-\mathrm{Mo}-\mathrm{Si}-\mathrm{B}(\bullet)$ 多晶合 金的显微硬度 $H$ 、与平均晶粒尺寸 $\bar{D}$ 的关系

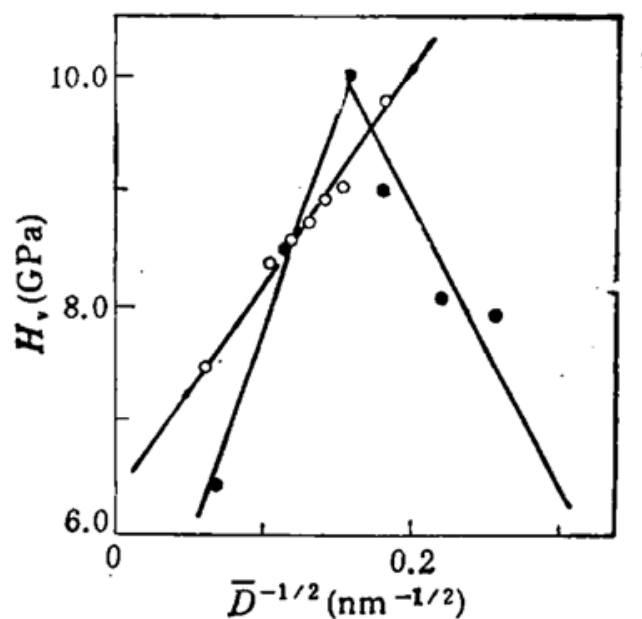

图 $4 \mathrm{Fe}-\mathrm{Cu}-\mathrm{Si}-\mathrm{B}(0)$ 及 $\mathrm{Fe}-\mathrm{Mo}-\mathrm{Si}-\mathrm{B}(\bullet)$ 多晶 合金的 $H_{v}-\bar{D}^{-\frac{1}{2}}$ 图

Hall-Petch 关系是描述多晶材料屈服应力与晶粒尺寸之间的常用表达式. 该式亦可写 成:

$$
H_{v}-H_{v_{0}}+f^{\prime} \cdot \bar{D}^{-\frac{1}{2}},
$$

将图 1、图 3 的结果绘成图 4. 显然, 对于 $\mathrm{Fe}-\mathrm{Cu}-\mathrm{Si}-\mathrm{B}$ 合金来说, $H_{、}$ 与 $\bar{D}^{-\frac{1}{2}}$ 之间为线性关 系,可表示为

$$
H_{\mathrm{v}}(\mathrm{GPa})-6.30(\mathrm{GPa})+19.6\left(\mathrm{GPa} \cdot \mathrm{nm}^{\frac{1}{2}}\right) \bar{D}^{-\frac{1}{2}}\left(\mathrm{~nm}^{-\frac{1}{2}}\right) \text {. }
$$

:而对于 $\mathrm{Fe}-\mathrm{Mo}-\mathrm{Si}-\mathrm{B}$ 合金而言, $H_{v}-\bar{D}$ 关系则为

$$
H_{\eta}(\mathrm{GPa})=\left\{\begin{array}{l}
4.28(\mathrm{GPa})+36.2\left(\mathrm{GPa} \cdot \mathrm{nm}^{\frac{1}{2}}\right) \cdot \bar{D}^{-\frac{1}{2}}\left(\mathrm{~nm}^{-\frac{1}{2}}\right), \bar{D}>45 \mathrm{~nm}, \\
13.75(\mathrm{GPa})-23.77\left(\mathrm{GPa} \cdot \mathrm{nm}^{\frac{1}{2}}\right) \cdot \bar{D}^{-\frac{1}{2}}\left(\mathrm{~nm}^{-\frac{1}{2}}\right), \bar{D}<45 \mathrm{~nm} .
\end{array}\right.
$$

即在 $\bar{D}<45 \mathrm{~nm}$ 范围内, 合金的显微硬度 $H$ 、 与平均晶粒尺寸 $\bar{D}$ 之间具有反 Hall-Petch 关系. 因此, Hall-Petch 关系在 $\mathrm{Fe}-\mathrm{Mo}-\mathrm{Si}-\mathrm{B}$ 小晶粒材料中并不完全适用. 比较图 4 中两 组合金的结果可知, 微量过渡元素 $\mathrm{Cu}, \mathrm{Mo}$ 对 $\mathrm{Fe}-\mathrm{Si}-\mathrm{B}$ 系列纳米晶合金的维氏硬度 $H$, 及其 随晶粒尺寸的变化规律有很大的影响.

$X$ 射线衍射 (XRD) 结果表明: 在 783-913K 范围内等温退火时, $\mathrm{Fe}-\mathrm{Cu}-\mathrm{Si}-\mathrm{B}$ 合金的 晶化产:物为 $\alpha-\mathrm{Fe}(\mathrm{Si})$ 固溶体 (bcc) 及 $\mathrm{Fe}_{2} \mathrm{~B}$ 化合物 (bct). 而 $\mathrm{Fe}-\mathrm{Mo}-\mathrm{Si}-\mathrm{B}$ 合金在 833- 
$913 \mathrm{~K}$ 范围内遈火时, 晶化相则为, $\alpha-\mathrm{Fe}(\mathrm{Si}, \mathrm{Mo})$ 固溶体 (bcc) 及 $(\mathrm{Fe}, \mathrm{Mo})_{3} \mathrm{~B}(\mathrm{bct}),(\mathrm{Fe}$. $\mathrm{Mo})_{23} \mathrm{~B}_{6}(\mathrm{fcc}), \mathrm{Fe}_{2} \mathrm{~B}(\mathrm{bct})$ 硼化物, 且随着温度的升高, ( $\left.\mathrm{Fe}, \mathrm{Mo}\right)_{3} \mathrm{~B}$ 和 $(\mathrm{Fe}, \mathrm{Mo})_{23} \mathrm{~B}_{6}$ 相逐渐转 化为 $\alpha-\mathrm{Fe}$ 和 $\mathrm{Fe}_{2} \mathrm{~B}$, 到 $1023 \mathrm{~K}$ 时两种亚稳相均已消失. 由此可知, $\mathrm{Cu}, \mathrm{Mo}$ 对两组合金力学 性能的影响,可能是通过影响非晶合金的晶化产物及晶化相的高温转变来实现的.

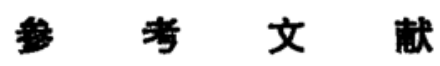

(1) Chokski, A. H., Rosen, A., Karch, J. et al, Scr. Meiall., 1989, 23(10): 1679-1684.

[ 2 ] Lu, K., Wei, W. D., Wang, J. T., Scr. Metall, 1991, 24(12): 2319-2323.

[ 3 ] Lu, K., Wang, J. T., Wei, W. D., J. Appl. Phys, i991, 69(1): 522-524. 